\title{
Estudo do estresse do enfermeiro com dupla jornada de trabalho em um hospital de oncologia pediátrica de Campinas*
}

\author{
STUDY ON THE STRESS OVER THE NURSE WHO WORKS IN TWO SHIFTS AT A PEDIATRIC ONCOLOGY \\ HOSPITAL IN CAMPINAS
}

\section{ESTUDIO DEL ESTRÉS DEL ENFERMERO CON DOBLE JORNADA DE TRABAJO EN UN HOSPITAL DE ONCOLOGIA PEDIÁTRICA DE CAMPINAS}

\section{Roberta Cova Pafaro', Milva Maria Figueiredo De Martino²}
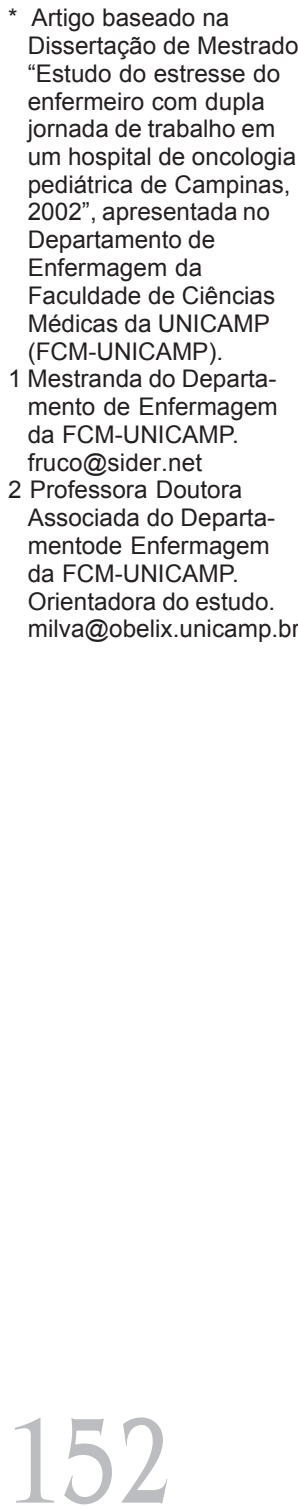

Rev Esc Enferm USP 2004; 38(2):152-60.

\author{
RESUMO \\ Trata-se de um estudo que teve \\ como objetivo investigar a \\ presença e o nivel de estresse \\ emocional, os sintomas fisicos \\ e psicológicos, a intensidade do \\ estresse e enfermeiros que \\ fazem dupla jornada de \\ trabalho comparados aos que \\ não fazem, num hospital de \\ oncologia pediátrica de \\ Campinas. A população foi \\ composta por 33 enfermeiros, \\ sendo 24 em regime de dupla \\ jornada e 9 em jornada única. \\ Na metodologia foi utilizado o \\ Inventario de Sintoma de \\ Stress LIPP e a Escala \\ Analógica Visual. Os \\ resultados permitiram \\ constatar que os enfermeiros \\ classificados quanto as fases \\ de estresse encontravam-se na \\ fase de resistência, com \\ referencia aos niveis de \\ estresse os mesmos \\ encontravam-se no nível médio \\ de estresse, houve \\ predominância dos sintomas \\ psicológicos e os enfermeiros \\ com dupla jornada estavam \\ mais estressados em relação \\ aos com jornada única.
}

\section{PALAVRAS-CHAVE}

Estresse.

Enfermeiros.

Trabalho em turnos.

\section{ABSTRACT}

This is a study that aimed investigating the presence and level of emotional stress, the physical and psychological symptoms, the intensity of stress and nurses who have two work shifts compared to the ones who don't have it at a pediatric oncology hospital in Campinas. The population was made of 33 nurses in which 24 work in two shifts and 9 work in just one. In the methodology, it was used the LIPP Stress Symptom Inventory and the Visual Analogical Scale. The results enabled us to confirm that nurses classified regarding to their stress phases were on the resistance phase, regarding to the stress level they were on the average stress level. There was a predominance of psychological symptoms and nurses who had two work shifts were more stressed than the ones with only one shift.

\section{KEYWORDS}

Stress.

Nurses.

Shift work.

\section{RESUMEN}

Se trata de un estudio que tuvo como objetivo investigar presencia y nivel de estrés emocional, sintomas fisicos y psicológicos, intensidad del estrés y enfermeros que realizan doble jornada de trabajo comparados a los que no lo hacen, en un hospital de oncología pediátrica de Campinas. La población estuvo compuesta por 33 enfermeros, de los cuales 24 en régimen de doble jornada y 9 en jornada única. En la metodología fue utilizado el Inventario de Síntoma de Stress LIPP y la Escala Analógica Visual. Los resultados permitieron constatar que los enfermeros clasificados, en relación a las fases de estrés, se encontraban en la fase de resistencia; con referencia a los niveles de estrés los mismos se encontraban en el nivel medio de estrés; hubo predominio de los síntomas psicológicos y los enfermeros con doble jornada estaban más estresados que los de jornada única.

\section{PALABRAS CLAVE}

Estrés.

Enfermeros.

Trabajo por turnos. 


\section{INTRODUÇÃO}

Sabe-se, hoje em dia, que o estresse é um dos fatores responsáveis por alterações do estado de saúde e de bem-estar do indivíduo que podem levar à doença e à morte. Por outro lado, têm-se multiplicado os esforços de pesquisa de especialistas e de instituições no sentido de propor mecanismos que visem controlar os aspectos negativos no trabalho. Essa necessidade de ação passou a ser particularmente visível no campo do controle do estresse, por ter sido provada a possibilidade de se prevenir a morbidade e impedir a mortalidade ocasionada por ele.

A palavra stress primeiramente utilizada na física, indicando o desgaste sofrido por materiais expostos a pressões ou forças ${ }^{(1)}$.

A palavra stress, derivada do latim, foi utilizada pela primeira vez no sentido psicológico no século XVIII (2). Porém, foi inicialmente usada na área da saúde por Hans Selye, na época estudante de medicina, em 1926, ao perceber que muitas pessoas sofriam de varias doenças físicas e apresentavam algumas queixas em comum como, fadiga, hipertensão, desânimo e falta de apetite. Em 1936, já então endocrinologista, introduziu o termo stress para designar uma síndrome produzida por vários agentes nocivos. Enfatiza a resposta não específica do organismo a situações que não o debilitam, enfraquecendo e levando o organismo a adoecer.

Quando publicou suas descobertas sobre os fenômenos do estresse, houve interesse médico mundial pelo assunto e vários pesquisadores puderam relacionar grande parte de seus achados de pesquisas com o esquema organizado por ele ${ }^{(3)}$.

O estresse pode ser definido como um desgaste geral do organismo, causado pelas alterações psicofisiológicas que ocorrem quando o indivíduo é forçado a enfrentar situações que o irritem, excitem, amedrontem, ou mesmo que o façam imensamente feliz ${ }^{(4)}$.

O estímulo que inicia uma reação de estresse é chamado de estressor. Um estímulo pode se tornar estressor em função da interpretação cognitiva ou do significado que o indivíduo atribui. Ou seja, algumas vezes, a interpretação que se dá a certos eventos é que os tornam estressantes e essa interpretação é o resultado da aprendizagem que ocorre durante o processo de nossa vida. Há situações em que o evento é intrinsecamente estressante independentemente da interpretação, como no caso de frio ou calor excessivo, dor, etc. $\mathrm{O}$ estressor pode ser algo negativo e também positivo que emocione a pessoa de modo marcante.

Atualmente o estresse significa pressão, insistência e estar estressado significa estar sob pressão ou estar sob ação de um determinado estímulo insistente ${ }^{(5)}$. Tem sido considerado como um dos problemas que mais freqüentemente age sobre o ser humano, interferindo na homeostase de seu organismo devido à grande quantidade de tensões que enfrenta diariamente.

A vulnerabilidade dos indivíduos ao estresse depende da sua habilidade para lidar com os eventos estressores. Não só estes, mas a maneira como o indivíduo lida com eles é fundamental para que se desenvolva um quadro de estresse. Uma vez que o modo de reagir a estímulos é um produto da aprendizagem, o contrário também pode ocorrer e é possível desaprender certas reações inadequadas e estressoras em potencial $^{(6)}$.

Os sinais e sintomas que ocorrem com maior freqüência são do nível físico como: aumento da sudorese, nó no estômago, tensão muscular, taquicardia, hipertensão, aperto da mandíbula e ranger de dentes, hiperatividade, mãos e pés frios, náuseas. Em termos psicológicos, vários sintomas podem ocorrer como: ansiedade, tensão, angústia, insônia, alienação, dificuldades interpessoais, dúvidas quanto a si próprio, preocupação excessiva, inabilidade de concentrar-se em outros assuntos que não o relacionado ao estressor, dificuldades de relaxar, tédio, ira, depressão, hipersensibilidade emotiva ${ }^{(4)}$.

Em estudos anteriores observou-se alterações nas glândulas supra-renais (dilatação do córtex), no timo (involução), no baço, no estômago (úlcera) e em muitas outras estruturas linfáticas do corpo $^{(7)}$. Identificou algo como uma única reação não específica do corpo a qualquer tipo de lesão infligida. A esse conjunto de respostas não específicas denominou Síndrome de Adaptação Geral (SAG). Verificou, após um tempo, que este conjunto evoluía de acordo com três fases, que seriam a reação de Alarme (RA), a fase de Resistência (FR) e a fase de Exaustão (FE).
Estudo do estresse do enfermeiro com dupla jornada de trabalho em um hospital de oncologia pediátrica de Campinas 
Estabeleceu três pontos fixos como parte da Síndrome: a supra-renal, o timolinfático e as alterações intestinais.

De acordo com esta teoria, as manifestações de estresse envolvem três fases que, em conjunto, caracterizam a Síndrome de Adaptação Geral:

\section{Estágio de defesa ou alarme}

Este estágio é caracterizado no organismo através do sistema nervoso central que percebe a situação de tensão e o hipotálamo estimula a hipófise, levando-a a aumentar a secreção do hormônio adrenocorticotrópico (ACTH). Este, por sua vez, estimula as supra-renais a aumentarem a produção de adrenalina e corticóides. Jogadas na circulação sistêmica rapidamente, estas substâncias chegam a todas as células do organismo. Estes hormônios são essencialmente úteis e, juntamente com as reações do sistema nervoso central e com outros componentes químicos, constituem a defesa do organismo contra o estresse. Se forem, porém descarrilados em sua produção podem causar doenças. Como exemplo, uma série de reações complexas ocorrem: a produção de hormônios aumenta; a respiração acelera; os batimentos cardíacos aumentam de freqüência; os músculos ficam tensos ${ }^{(1)}$.

\section{Fase de Resistência}

Ocorre quando o estressor perdura por um período muito prolongado, havendo um aumento da capacidade de resistência do organismo. Há plena adaptação ao estressor, ficando a atividade mais intensa em função do sistema parassimpático, possuindo o efeito de desmobilizar o corpo, pois este abaixa novamente o nível de alerta. A respiração, os batimentos cardíacos, a circulação e a pressão arterial voltam gradativamente a seus níveis anteriores. Porém, havendo persistência do estresse, o nível de resistência vai diminuindo e inicia-se o estágio de exaustão ${ }^{(8)}$.

\section{Fase de exaustão ou esgotamento}

Nessa fase, os sintomas da fase de alerta reaparecem mais acentuados e outros desenvolvem-se, tornando o organismo mais suscetível a doenças. Caracteriza-se pela incapacidade dos mecanismos responsáveis pela busca da adaptação do organismo aos efeitos dos estressores permanecerem por tempo prolongado. O estresse torna-se intenso e, conseqüentemente, esgotando toda a energia adaptativa do organismo ${ }^{(8)}$.

Exaustão é a quebra do equilíbrio do organismo e está associada a uma série de doenças como hipertensão arterial, depressão, ansiedade, problemas sexuais e dermatológicos, como psoríase, vitiligo, urticárias e alergia, além do infarto e até da morte súbita.

Embora inicialmente tenha-se identificado somente três fases do estresse (alerta, resistência e exaustão), em estudo desenvolvido posteriormente, identificou-se uma quarta fase tanto clínica como estatisticamente denominada de quase-exaustão por se encontrar entre a fase de resistência e a da exaustão, caracteriza-se por um enfraquecimento da pessoa que não mais está conseguindo adaptar-se ou resistir ao estressor $^{(9)}$. As doenças começam a surgir, porém, ainda não são tão graves como na fase da exaustão. Embora apresentando desgaste e outros sintomas, a pessoa ainda consegue trabalhar na sociedade até certo ponto, ao contrário do que ocorre na de exaustão, quando a pessoa não consegue, na maioria das vezes, trabalhar ou concentrarse. Desse modo, a fase de resistência referese à primeira parte do conceito de resistência de Selye enquanto a fase de quase-exaustão refere-se à parte final desta quando a resistência da pessoa está realmente se exaurindo.

Um alto nível de estresse contínuo pode gerar um quadro de esgotamento físico e emocional caracterizado por pessimismo, imagens negativas de si mesmo, atitudes desfavoráveis em relação ao trabalho, mais conhecidas como Síndrome de Burnout.

Esta Síndrome pode ser definida como sendo a que acomete aqueles profissionais cujas profissões têm relação direta com as pessoas, e que estão expostos a um estresse crônico. A Síndrome de Burnout tem como traços característicos o desgaste emocional, a despersonalização e a reduzida satisfação pessoal ou sentimento de incompetência do indivíduo. O fenômeno do Burnout pode ser caracterizado como uma síndrome de má adaptação psicológica, psicofisiológica e de reações comportamentais inadequadas, a uma forma específica de estresse ocupacional. Esta definição permite uma visão compreensiva de como a realidade emocional do indivíduo associada à institucional, pode 
afetar os profissionais que trabalham nos serviços sociais ${ }^{(10-11)}$.

Os principais sintomas psicossomáticos associados referem-se ao aparecimento de cefaléias, tensões musculares, hipertensão arterial e outros. As alterações mais freqüentemente observadas na conduta são: o absenteísmo ao trabalho, a conduta violenta, a incapacidade para relaxar, além do aumento do consumo de tabaco, álcool, fármacos, entre outros. Já na dimensão emocional: impaciência, irritabilidade, distanciamento afetivo, ansiedade e redução da capacidade de elaboração de juízos podem ser observados. O conjunto desses sintomas influirá diretamente no inter-relacionamento pessoal e, conseqüentemente, na relação profissional.
Estudo do estresse do enfermeiro com dupla jornada de trabalho em um hospital de oncologia pediátrica de Campinas

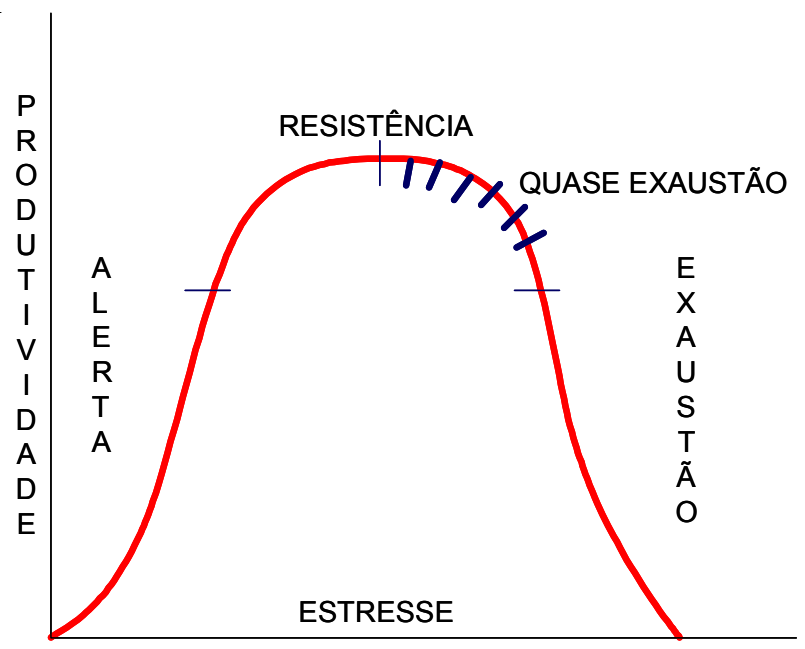

Figura 1 - Produtividade do indivíduo em relação as fases do estresse (Pafaro, 2002)

O estresse relaciona-se diretamente com a produtividade do indivíduo, uma pessoa que não tem estresse não produz, pois ela acaba não produzindo adrenalina, tornandose uma pessoa apática, desanimada, totalmente improdutiva. Portanto quanto mais estresse, maior a produção de adrenalina, conseqüentemente maior a produtividade. Quando o estresse extrapola o limite do indivíduo, sua produtividade começa a diminuir, e ele pode contrair doenças e até mesmo morrer $^{(9)}$. (Figura 1)

As diferenças entre o estresse positivo e o negativo caracteriza-se como ${ }^{(13)}$ : EUSTRESS - o estresse de natureza positiva, a pessoa tenciona-se, atingindo um nível ideal de esforço e é realimentada pelos resultados; e DISTRESS - o estresse que adoece, que está relacionado à sobrecarga, no qual ocorre a ativação crônica e repetida do eixo hipotálamo-hipófise-adrenal, no qual a constante elevação dos hormônios origina alterações patológicas.

\section{Características gerais da dupla jornada de trabalho em enfermagem}

A dupla jornada de trabalho, faz-se necessária aos trabalhadores de enfermagem devido à situação econômica da área da saúde, aos baixos salários insuficientes para o sustento da família, o que os leva a procurar novas fontes de renda. $\mathrm{Na}$ realidade, necessitam enfrentar dupla atividade, o que pode interferir em alguns aspectos referentes à qualidade de vida do trabalhador.

Os múltiplos papéis assumidos pela maioria das mulheres que exercem uma atividade profissional tendem a remetê-las a determinadas situações em que se sentem impotentes e frustradas por não conseguirem conciliar seus inúmeros afazeres. A sobrecarga de trabalho, com jornadas duplas ou triplas pode conduzir a mulher ao stress emocional, considerando que sua inserção no mercado de trabalho não a desvinculou das tarefas domésticas e da educação dos filhos resultando num acúmulo de atribuições ${ }^{(14)}$.

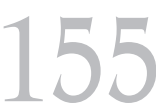

Rev Esc Enferm USP 2004; 38(2):152-60 


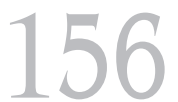

Rev Esc Enferm USP 2004; 38(2):152-60.
O trabalho em turnos é uma característica do exercício da enfermagem, sendo obrigatório uma vez que a assistência é prestada durante as 24 horas do dia, nos 7 dias da semana, ininterruptamente. Essa condição obriga que a assistência ocorra à noite, nos finais de semana, nos feriados, períodos estes utilizados por outros trabalhadores para dormir, descansar, usufruir do lazer e do convívio social e familiar $^{(15-16)}$.

De uma maneira geral, o serviço de enfermagem é composto por pessoas do sexo feminino, fazendo com que estas tenham uma carga de trabalho dupla, principalmente quando se somam as atividades domésticas ou quando são obrigadas a trabalharem em outro emprego ${ }^{(15)}$.

A intensidade da vivência que o enfermeiro hospitalar experimenta no seu cotidiano the exige uma contínua e profunda mobilização de energia adaptativa que, por vários motivos, pode não estar disponível ou pode não ser suficiente para evitar o estresse ${ }^{(17)}$.

Os riscos para a saúde relacionados com o trabalho dependem do tipo de atividade profissional e das condições em que ela é desempenhada. Os serviços de saúde, e de um modo particular os hospitais, proporcionam aos seus funcionários condições de trabalho reconhecidamente piores do que as verificadas na grande maioria dos outros setores de atividade. Para além dos acidentes de trabalho e das doenças profissionais propriamente ditas, a atividade de enfermagem contribui de forma decisiva para a ocorrência de doenças relacionadas com o trabalho. Os enfermeiros encontram-se expostos do ponto de vista etiológico aos fatores de risco de natureza física, química, biológica e psicossocial; que se fazem sentir com grande intensidade e justificam a inclusão da profissão de enfermagem no grupo das profissões desgastantes ${ }^{(18)}$.

A enfermagem é uma profissão consi-derada como potencialmente estressante. De fato, em 1988, a enfermagem foi classificada pela Health Education Authority ${ }^{(19)}$, como a quarta profissão mais estressante no setor público.

Diante do exposto, foi nosso objetivo nesta pesquisa verificar a presença de sintomas físicos e psicológicos de estresse nos enfermeiros; através da auto-avaliação verificar a intensidade do estresse emocional e verificar se há diferença na presença de estresse em enfermeiros com jornada dupla e única de trabalho.

\section{METODOLOGIA}

Do total de 35 enfermeiros, participaram deste estudo 33, pois um encontrava-se em licença-gestação e outro em licença médica. A faixa etária estava compreendida entre 23 a 51 anos. Quanto ao sexo, 28 eram do sexo feminino e 5 masculino.

A amostra foi, portanto, composta de 33 sujeitos sendo que 24 faziam dupla jornada de trabalho e 9 trabalhavam sem dupla jornada. Os turnos eram fixos com os seguintes horários: período da manhã das 8 às 14 horas; vespertino, das 14 às 20 horas $\mathrm{e}$ noturno, das 20 às 8 horas.

Os instrumentos utilizados para a coleta de dados foram: Termo de consentimento livre e esclarecido; Ficha de identificação; Inventário de Sintomas de stress I.S.S. Lipp ${ }^{(9)}$; Escala Analógica Visual (E.A.V. uma adaptação da escala usada por Darini ${ }^{(20)}$ ).

Para realizar a coleta de dados, primeiramente foi feito um levantamento do total de enfermeiros que faziam ou não a dupla jornada de trabalho nos diferentes turnos do Hospital de Oncologia Pediátrica de Campinas.

Após a confirmação voluntária de participação do sujeito, a pesquisadora iniciou a coleta de dados da seguinte forma: inicialmente entregou o Termo de Consentimento para ser assinado. Em seguida, foi aplicado o Inventário de Sintomas de Stress Lipp (ISSL), foram lidas as questões e anotadas as respostas. Na seqüência, foi solicitado aos participantes que assina-lassem a Escala Analógica Visual (EAV). Os testes psicológicos utilizados foram avaliados e interpretados por uma psicóloga. Os resultados obtidos foram analisados através do Teste Exato de Fisher para verificar a existência de associação entre grupos com relação às variáveis categóricas. Os horários da coletas foram manhã, tarde e noite, nos meses de agosto a setembro de 2001 .

O projeto foi aprovado pela Comissão de Ética em Pesquisa da Faculdade de Ciências Médicas da UNICAMP, homologado em 18 de setembro de 2001. 


\section{RESULTADOS E DISCUSSÃO}

Com relação aos que faziam dupla jornada, 70,84\% apresentaram estresse e $29,16 \%$ não acusaram sua presença. Para os sujeitos do grupo sem dupla jornada, $55,56 \%$ apresentaram estresse e $44,44 \%$ não acusaram a presença de estresse. Embora o teste Estatístico Exato de Fisher utilizado não tenha demonstrado diferença significativa quanto ao tipo de jornada em relação à presença de estresse, os valores percentuais acusaram diferença que pode ser justificada. (Tabela 1)

Dos sujeitos pertencentes ao grupo com dupla jornada conforme a Tabela 2, 37,50\% apresentaram sintomas psicológicos, $29,17 \%$ não apresentaram sintomas, 20,83\% apresentaram sintomas físicos e 12,50\% apresentaram sintomas físicos e psicológicos. Para o grupo sem dupla jornada, 33,33\% apresentaram sintomas psicológicos, 44,44\% não apresentaram sintomas, $11,11 \%$ apresentaram sintomas físicos e $11,11 \%$ apresentaram sintomas físicos e psicológicos. Portanto pode-se observar que, dentre os sintomas, houve predominância dos psicológicos, embora o Teste de Fisher não mostrou diferença significativa quando comparamos as variáveis com dupla e sem dupla jornada de trabalho, em relação à avaliação dos sintomas físicos e psicológicos.

Tabela 1 - Avaliação dos sujeitos quanto à presença ou não de estresse de acordo com o tipo de jornada (Campinas, 2001)

\begin{tabular}{lcccccc}
\hline \multirow{2}{*}{$\begin{array}{c}\text { Variáveis } \\
\text { (tipo de jornada) }\end{array}$} & \multicolumn{2}{c}{ Com estresse } & \multicolumn{2}{c}{ Sem estresse } & \multicolumn{2}{c}{ Total } \\
\cline { 2 - 7 } & Freq. & $\mathbf{\%}$ & Freq. & $\mathbf{\%}$ & Freq. & $\%$ \\
\hline Com dupla jornada & 17 & 70,84 & 7 & 29,16 & 24 & 100,00 \\
Sem dupla jornada & 5 & 55,56 & 4 & 44,44 & 9 & 100,00 \\
\hline Total & 22 & 66,66 & 11 & 33,34 & 33 & 100,00 \\
\hline
\end{tabular}

Estatística prob Fisher'Exact test $\mathrm{p}=0,438$

Tabela 2 - Avaliação dos sintomas físicos e psicológicos conforme o tipo de jornada da população estudada (Campinas, 2001)

\begin{tabular}{lcccccccccc}
\hline & \multicolumn{7}{c}{ Sintomas } \\
\cline { 2 - 10 } Variáveis & \multicolumn{1}{c}{ Físicos } & $\begin{array}{c}\text { Físicos e } \\
\text { psicológicos }\end{array}$ & Psicológicos & $\begin{array}{c}\text { Ausência } \\
\text { de sintomas }\end{array}$ & Total \\
\cline { 2 - 11 } & Freq & $\%$ & Freq & $\%$ & Freq & $\%$ & Freq & $\%$ & Freq & $\%$ \\
\hline $\begin{array}{l}\text { Com dupla } \\
\text { jornada } \\
\begin{array}{l}\text { Sem dupla } \\
\text { jornada }\end{array}\end{array}$ & 5 & 20,83 & 3 & 12,50 & 9 & 37,50 & 7 & 29,17 & 24 & 100,0 \\
\hline Total & 1 & 11,11 & 1 & 11,11 & 3 & 33,33 & 4 & 44,44 & 9 & 100,0 \\
\hline
\end{tabular}

Estatística prob Fisher'Exact test $\mathrm{p}=0,8927$

Conforme os dados apresentados na Tabela 3 , do grupo com dupla jornada, $45,83 \%$ apresentou resultados relativos ao nível médio de estresse e, para os níveis alto e baixo de estresse $37,50 \%$ e $16,67 \%$, respectivamente.

Com relação aos sujeitos pertencentes ao grupo sem dupla jornada foram classificados $55,55 \%$ com baixo nível de estresse. Quanto à classificação dos níveis de estresse médio e alto, obtivemos os valores de $22,22 \%$ para ambos.
Verificou-se que a maioria dos sujeitos analisados, com e sem dupla jornada de trabalho, obtiveram a classificação para o nível médio de estresse.

Não houve diferença estatisticamente significativa pelo teste de Fisher, quando se comparou as variáveis com dupla e sem dupla jornada de trabalho, em relação ao estresse subjetivo, isto é, avaliado pelos próprios sujeitos através do EAV.
Estudo do estresse do enfermeiro com dupla jornada de trabalho em um hospital de oncologia pediátrica de Campinas 
Visual - EAV (Campinas, 2001)

\begin{tabular}{lccccccccc}
\hline \multirow{2}{*}{ Variáveis } & \multicolumn{8}{c}{ Níveis de estresse } \\
\cline { 2 - 10 } & \multicolumn{2}{c}{ Baixo } & \multicolumn{2}{c}{ Médio } & \multicolumn{2}{c}{ Alto } & \multicolumn{2}{c}{ Total } \\
\cline { 2 - 10 } & Freq. & $\%$ & Freq. & $\%$ & Freq. & $\%$ & Freq. & $\%$ \\
\hline Com dupla jornada & 4 & 16,67 & 11 & 45,83 & 9 & 37,50 & 24 & 100,00 \\
Sem dupla jornada & 5 & 55,55 & 2 & 22,22 & 2 & 22,22 & 9 & 100,00 \\
\hline Total & 9 & 27,27 & 13 & 39,4 & 11 & 33,33 & 33 & 100,00 \\
\hline
\end{tabular}

Estatística prob Fisher'Exact test.p=0,133

Os sujeitos do grupo relacionados na Tabela 4, com dupla jornada de trabalho obtiveram como resultado: $62,50 \%$ na fase de resistência; $29,16 \%$ a ausência de estresse e apenas $8,34 \%$ encontraram-se na fase de quase exaustão e exaustão.

Para o grupo sem dupla jornada, classificados conforme as fases de estresse $55,56 \%$ estavam na fase de resistência enquanto $44,44 \%$ demonstraram ausência de estresse; quanto à fase de quase exaustão e exaustão não houve classificação.

Nenhum sujeito da pesquisa foi classificado como pertencente à fase de alerta.
Não houve diferença significativa pelo teste de Fisher quando se comparou as variáveis com dupla e sem dupla jornada de trabalho em relação às fases de estresse ISS -Lipp ${ }^{(9)}$.

Entretanto, podemos verificar que ao somarmos $62,50 \%$ dos sujeitos que se encontravam na fase de resistência aos $8,34 \%$ dos sujeitos que estavam na fase de exaustão ou quase exaustão, totatizase um percentual de $70,84 \%$ de níveis de estresse para a população do grupo com dupla jornada, enquanto que $55,56 \%$ de níveis de estresse para o grupo sem dupla jornada.

Tabela 4 - Fases de estresse obtidas através do Inventário de Sintomas de Stress LIPP (Campinas, 2001)

\begin{tabular}{|c|c|c|c|c|c|c|c|c|c|c|}
\hline \multirow{3}{*}{ Variáveis } & \multicolumn{10}{|c|}{ Fases de estresse } \\
\hline & \multicolumn{2}{|c|}{ Ausência } & \multicolumn{2}{|c|}{ Alerta } & \multicolumn{2}{|c|}{ Resistência } & \multicolumn{2}{|c|}{$\begin{array}{c}\text { Quase } \\
\text { exaustão e } \\
\text { exaustão }\end{array}$} & \multicolumn{2}{|c|}{ Total } \\
\hline & Freq & $\%$ & Fre & $\%$ & Freq & $\%$ & Freq & $\%$ & Freq & $\%$ \\
\hline Com dupla jornada & 7 & 29,16 & - & - & 15 & 62,50 & 2 & 8,34 & 24 & 100,0 \\
\hline Sem dupla jornada & 4 & 44,44 & - & - & 5 & 55,56 & - & - & 9 & 100,0 \\
\hline Total & 11 & 33,34 & - & - & 20 & 60,6 & 2 & 6,06 & 33 & 100,0 \\
\hline
\end{tabular}

Estatística prob Fisher. Exact test $\mathrm{p}=0,834$

\section{CONCLUSÕES}

Os resultados obtidos em nossa pesquisa evidenciaram que, com relação às características gerais da população estudada, houve predomínio do sexo feminino $(84,84 \%)$.

Observou-se também, que os mais jovens e solteiros pertenciam ao grupo sem dupla jornada.

Em relação aos níveis de estresse, encontrou-se que do total de 33 enfermeiros,
$22(66,67 \%)$ estavam com estresse e 11 $(33,33 \%)$ não o apresentaram, ou seja, a maioria estava estressada.

Observou-se que os sujeitos classificados quanto às fases de estresse de ambos os grupos estavam na fase de resistência, avaliados através do ISS- Lipp ${ }^{(9)}$.

Com referência aos níveis de estresse, a maioria de ambos os grupos encontrava-se no nível médio de estresse, avaliados através do $\mathrm{EAV}^{(20)}$. 
Ao analisar os sintomas físicos e psicológicos, houve uma predominância dos sintomas psicológicos.

Ao se comparar os níveis de estresse através do ISS -Lipp ${ }^{(9)}$ e $\mathrm{EAV}^{(20)}$ em relação aos grupos com dupla e sem dupla jornada, estatisticamente não houve diferença significativa.

Entretanto, as porcentagens demonstraram o contrário, pois obtivemos $70,84 \%$ para o grupo com dupla jornada, que estavam estressados em comparação a 55,56\% pertencentes ao grupo sem dupla jornada que também apresentaram estresse, conforme o ISS- Lipp ${ }^{(9)}$.

\section{CONSIDERAÇÕES FINAIS}

Sabemos que o estresse pode se originar de fontes externas ou internas. As externas são aquelas representadas pelo que nos acontece na vida ou pelas pessoas com as quais lidamos, isto é, trabalho em excesso ou desagradável, família em desarmonia, acidentes, morte, tensão, violência, medo, doença, etc.

As causas internas são aquelas que se referem a como pensamos, às crenças e aos valores que temos e como interpretamos o mundo ao nosso redor.

É fundamental descobrir a causa do problema e desenvolver estratégias de enfrentamento para lidar não só com o episódio presente, mas também com futuras ameaças de estresse excessivo.
O que gera estresse em uma pessoa é o modo como ela reage à situação. Essa reação, por sua vez, depende de variáveis pessoais como temperamento e experiências passadas além do modo como essas varáveis influen-ciam na interpretação que a pessoa faz dos fatos ${ }^{(4)}$.

As fontes internas de estresse têm início na infância, de acordo com as mensagens e os valores transmitidos pelos adultos responsáveis pela educa-ção da criança, principalmente por meio de seus comportamentos, uma vez que os mais marcantes na infância são os atos, que aos poucos são por ela assimilados e imitados.

O estresse pode vir a ser criado pela própria criança, de acordo com a sua aprendizagem social, seus pensamentos, tipo de personalidade e atitudes. Assim sendo, a família, em especial os pais, a escola, a comunidade e outras instituições, que a criança freqüenta com certa assiduidade, influenciam direta e indiretamente, levando-a a adquirir comportamentos que podem desencadear um estresse intenso. Portanto uma criança estressada poderá vir a ser um adulto fragilizado, vulnerável ao estresse. Talvez o que se possa dizer de mais relevante sobre os determinantes do estresse com origem no passado relaciona-se ao grau com que o indivíduo se expõe às demandas de seu meio, estando em condições de atendêlas e tendo sido dirigido no sentindo de lidar com sucessos e fracassos ${ }^{(21-22)}$.

É importante valorizar o sujeito, seu ambiente de trabalho e as especificidades de ambos.
Estudo do estresse do enfermeiro com dupla jornada de trabalho em um hospital de oncologia pediátrica de Campinas

\section{REFERÊNCIAS}

(1) Lima EDRP, Carvalho DV Estresse ocupacional. Rev nursing, 2000; 22:30-34.

(2) Spilberger C. Understanding stress and anxiety. New York: Haper e Row Publishers; 1979.

(3) Albrecht, K. O gerente e o estresse. Rio de Janeiro: J.Z.E;1990.

(4) Lipp MEN. O stress está dentro de você.São Paulo: Contexto; 2000
(5) Proença MI. Stress ocupacional e qualidade de vida do jornalista da mídia impressa diária. [dissertação]Campinas(SP): Faculdade de Psicologia da Pontifícia Universidade Católica de Campinas; 1998.

(6) Lipp MEN. Stress e suas implicações. Rev psicol,1984; 3(4):05-19.

(7) Selye H. Stress a tensão da vida. 2 nd ed. São Paulo: Ibrasa;1965. 
Roberta Cova Pafaro Milva Maria F De Martino
(8) Furlan V. Stress em mães de crianças portadoras do HIV.[dissertação]Campinas(SP): Faculdade de Psicologia da Pontifícia Universidade Católica de Campinas; 1997.

(9) Lipp MEN. Inventário de sintomas de stress para adultos de Lipp (ISSL). São Paulo: Casa do Psicólogo; 2000.

(10) Selligman-Silva. E. Psicopatologia e psicodinâmica no trabalho.In: Mendes R. Patologia do trabalho. Rio de Janeiro: Atheneu; 1995. p. 287-310.

(11) Wolfgang AP. Job stress in the health professions: a study of physicians, nurses, and pharmacists. Hosp Topics; 1988; 4: 24-28.

(12) Pafaro RC. Estudo de estresse do enfermeiro com dupla jornada de trabalho em um hospital de oncologia pediátrica de Campinas [dissertação]Campinas(SP): Faculdade de Ciências Médicas da Universidade Estadual de Campinas; 2002.

(13) Bernik, WM.D Stress: o assassino silencioso. Disponível em URL: http://www.epub. org.br/cm/n03/doenças/stress.htm $>(21 \mathrm{abr}$ 2000).

(14) Spíndola T. Mulher, mãe e... trabalhadora de enfermagem. Rev Esc Enferm USP 2000; 34(4):354-61

(15) De Martino MMF. Estudo da variabilidade circadiana da temperatura oral, ciclo vigíliasono e testes psicofisiológicos em enfermeiros de diferentes turnos de trabalho.[Tese] Campinas(SP): Instituto de Biologia da Universidade Estadual de Campinas, 1996.
(16) Martino MMF. Estudo da arquitetura do sono e características do ciclo vigília-sono em enfermeiras de diferentes turnos. [Livre Docência]Campinas(SP): Faculdade de Ciências Médicas da Universidade Estadual de Campinas; 2002.

(17) Chaves EC Stress e trabalho do enfermeiro: a influência de características individuais no ajustamento e tolerância do turno noturno. [Doutorado] São Paulo(SP): Instituto de Psicologia da Universidade de São Paulo;1994.

(18) Gaspar PJS. Enfermagem profisssão de risco e desgaste. Rev nursing 1997;109(3):23-24

(19) Copper CL, Mitchel S. Nursing and critically ill and dying. Hum Relations 1990; 43: 297-311.

(20) Darini JA, Dor crônica: comparação da intensidade da dor, depressão, ansiedade e estratégias de manejo entre três grupos de pacientes.[Dissertação]Campinas(SP): Pontifícia Universidade Católica de Campinas;1991.

(21) Lipp MEN. Crianças estressadas: causas, sintomas e soluções. Campinas: Papirus; 2000.

(22) Lipp MEN. Como enfrentar o stress. São Paulo: Ícone; 1998. 